\title{
You might owe your mother more than you think
}

\author{
Leticia De las Vecillas ${ }^{1}$, Ibon Eguiluz-Gracia ${ }^{2}$, and Mattia Giovannini ${ }^{3}$ \\ ${ }^{1}$ Hospital Universitario Marques de Valdecilla \\ ${ }^{2}$ IBIMA-Regional University Hospital of Málaga, UMA, Malaga \\ ${ }^{3}$ Anna Meyer Chidren's University Hospital
}

February 22, 2021

\section{News and Views: Groundbreaking Discoveries in Immunology}

Title: You might owe your mother more than you think

Authors: Leticia de las Vecillas ${ }^{1}$, Ibon Eguiluz-Gracia ${ }^{2}$, Mattia Giovannini ${ }^{3}$

${ }^{1}$ Allergy Department, Marqués de Valdecilla University Hospital-Instituto de Investigación Marqués de Valdecilla (IDIVAL), Santander, Spain. leticia.delasveci@gmail.com; ORCID 0000-0003-4969$5678^{2}$ Allergy Unit, ARADyAL, IBIMA-Hospital Regional Universitario de Malaga-UMA, Malaga, Spain. iboneguiluz@gmail.com; ORCID 0000-0002-3774-931X

${ }^{3}$ Allergy Unit, Department of Pediatrics, Meyer Children's University Hospital, Florence, Italy. mattia.giovannini@unifi.it; ORCID 0000-0001-9568-6882

Correspondence to : Leticia de las Vecillas, Allergy Department, Marques de Valdecilla University Hospital-Instituto de Investigacion Marques de Valdecilla (IDIVAL), Santander, Spain. Email: leticia.delasveci@gmail.com; ORCID 0000-0003-4969-5678. Mattia Giovannini, Allergy Unit, Department of Pediatrics, Meyer Children's University Hospital, Florence, Italy. mattia.giovannini@unifi.it; ORCID 00000001-9568-6882

Word count: 653

Number of tables: 0

Number of figures: 1

Keywords: IgE; mast cells; protection; Staphylococcusaureus; allergy; murine models; pediatrics, transplacental transfer, toxin hypothesis.

Abbreviations: CXCL1/2, chemokine (C-X-C motif) ligand 1/2; FceRI, high-affinity receptor for the Fc region of IgE; FcRN, neonatal Fc receptor; MC, mast cell; SA, Staphylococcus aureus ; sIgE, specific immunoglobulin E; Tfh13, T follicular helper 13; TNF, tumor necrosis factor.

Statement of conflict of interest: the authors declare no conflict of interest in relation to this work.

Statement of contribution : LV and MG conceptualized the article and wrote the manuscript. IEG supervised the work of the rest of the authors and reviewed the final version of the article.

Statement of funding : This work was supported by the Instituto de Salud Carlos III of the Spanish Ministry of Science and Competitiveness (grants co-funded by the European Regional Development Fund) through the research contract "Juan Rodes" for IEG (JR19/00029), and the program of Redes Temáticas de Investigación Colaborativa en Salud (RETICS): Asma, Reacciones Adversas y Alérgicas-ARADyAL (RD16/0006/0001). 
Ethical approval and trial registration: Not applicable.

Consent for publication: Not applicable.

Data sharing and data accessibility: Not applicable.

Acknowledgements: The authors want to thank Dr. Anna Globinska for Figure 1 design and Dr. JiménezSaiz for critical review of the manuscript.

Abstract: Not applicable.

Main text

Mast cell (MC) activation and the subsequent release of inflammatory mediators has been historically linked to allergic responses ${ }^{1}$. In this regard, allergen-specific immunoglobulin E (sIgE) is a potent activator of MCs through FceRI crosslinking after allergen ligation to the IgE bound to FceRI on the surface of MCs. This activation pathway of MCs leads to potentially life-threatening reactions such as anaphylaxis.

Nevertheless, MCs also respond to triggers other than sIgE. For example, venoms from different insects can drive MC degranulation in both antibody-dependent and -independent manners ${ }^{2}$, thus providing efficient innate and adaptive protection against toxic compounds. Moreover, healthy subjects can display IgE against toxins from Staphylococcus aureus (SA) (SA-sIgE) ${ }^{3}$. Therefore, MCs may also have a role in the adaptive protection against venoms or toxins.

Following this idea, the so-called toxin hypothesis ${ }^{4,5}$, Starkl et al.$^{6}$ investigated the contribution of MCs and SIgE to the host's resistance against SA. Using a mouse model, the authors showed that the epicutaneous inoculation of SA induces an early serum increase of functional SA-sIgG1/sIgE and total IgE. This is followed by an expansion of $\mathrm{T}$ follicular helper (Tfh) cells in the inguinal lymph nodes, especially of cell subsets directly involved in high-affinity IgE production such as $\mathrm{Tfh}_{13} 3^{7,8}$. SA toxins also induced peripheral CD4+ and CD8+ T-cell proliferation and IgE synthesis via monoclonal and polyclonal mechanisms in the absence of live bacteria. Interestingly, a single skin inoculation of SA suffices to mount a systemic immune that yields SA-sIgE. Upon subsequent infections, MCs from different organs release high amounts of bactericidal compounds and chemoattractants through SA-sIgE-mediated mechanisms. Some of these chemotactic factors (such as TNF and CXCL1/2) ${ }^{6}$ are involved in neutrophil recruitment, thus reducing bacterial load and improving SA clearance. Collectively, IgE immunity confers an evolutionary advantage which translates into a higher survival rate and a quicker recovery following severe SA infections, including pneumonia. Curiously, bactericidal compounds are also released when MCs are activated by antigens other than SA toxin, which generally confers potential antibacterial activities to allergic reactions.

In addition to the adulthood, sIgE might also exert protective roles from the embryological development. Msallam et al . have recently investigated the interactions between fetal MCs and maternal sIgE to shed light on their relationship in fetal priming of immune and allergic responses in mice and humans ${ }^{9}$. Mouse fetal MCs acquire a semi-mature phenotype according to FceRI and heparin expression, and increase in number during early development stages, which suggests that they might be functional before birth. Of note, the skin and the airways hosted significant mouse fetal MCs populations. Furthermore, the neonatal $\mathrm{Fc}$ receptor $(\mathrm{FcRN})$ can translocate maternal $\mathrm{IgE}$ to the fetus, thus allowing the sensitization of mouse fetal MCs. Intriguing, the authors also showed that maternal sIgE can induce degranulation of mouse fetal MCs, but this effect was progressively lost during the 6 weeks following the offspring's birth. Moreover, the authors partially replicated these findings in humans as they identified a subset of human fetal MCs that were phenotypically mature (based on FceRI and CD63) since the second trimester of pregnancy. Lastly, they showed that human fetal MCs can also be sensitized with $\operatorname{IgE}$ in utero .

Overall, these data suggest that the offspring of atopic mothers can become passively sensitized in utero , thus being able to experience allergic reactions during the perinatal period without previous contact with the allergen. This passive sensitization phenomenon event constitutes a novel and clinically-relevant concept in newborns of allergic mothers. On the other hand, fetal MCs sensitization with maternal IgE might provide 
temporary protection from toxins and venoms. This defence may be of paramount importance during the first weeks of life given that the offspring is still developing immunity. Future research should expand these findings and investigate how to apply them for the development of antibacterial and antivenom approaches without the risk of suffering allergic reactions. By and large, these findings contribute to expand the growing list of homeostatic and protective roles of $\operatorname{Ig} \mathrm{E}^{4,5,6,10}$.

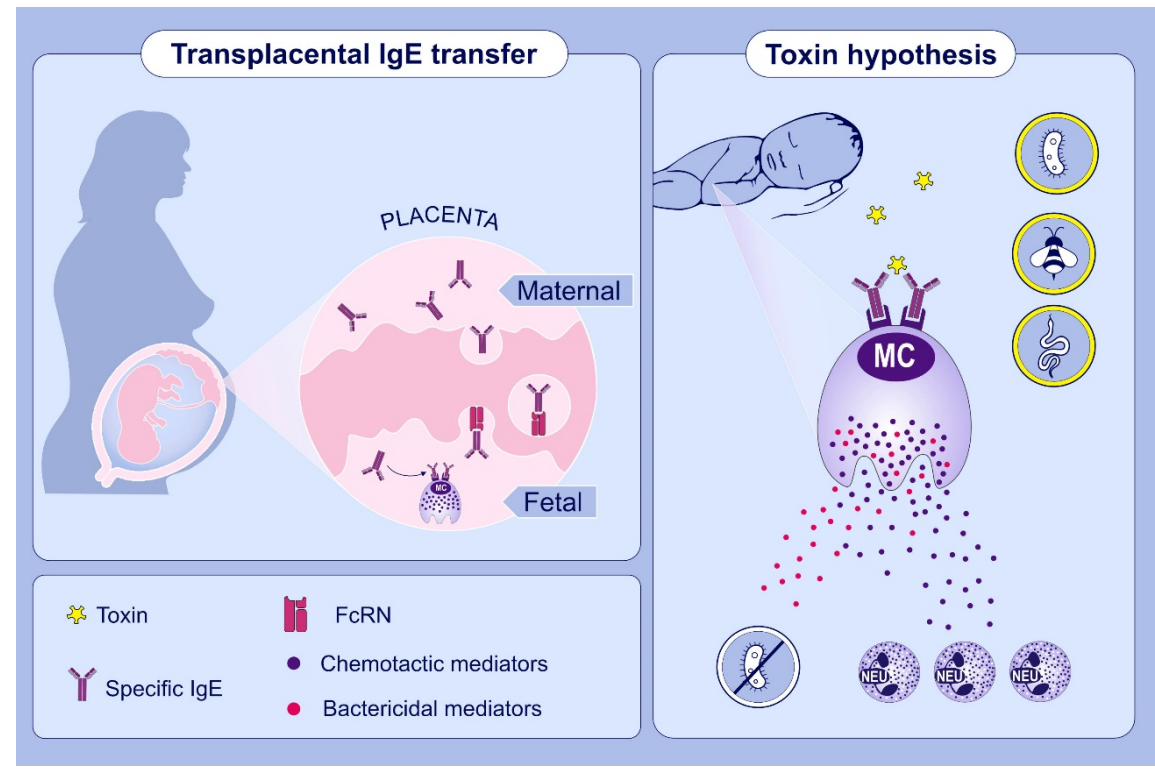

Figure 1. Scheme of IgE's vertical transmission and the sensitization of fetal MCs for allergen-specific degranulation by IgE of maternal origin through the neonatal Fc receptor (FcRN) (left side). Prenatal sensitization may include protective functions in the offspring described by the "Toxin hypothesis", in which mast cells confer adaptive protection against venoms and bacterial toxins (left side).

\section{References}

1. Galli SJ, Tsai M. IgE and mast cells in allergic disease. Nat Med. 2012;18:693-704.

2. Anderson E, Stavenhagen K, Kolarich D, Sommerhoff CP, Maurer M, Metz M. Human Mast Cell Tryptase Is a Potential Treatment for Snakebite Envenoming Across Multiple Snake Species. Front Immunol. 2018;9:1532. Available from: https://www.frontiersin.org/article/10.3389/fimmu.2018.01532

3. Sintobin I, Siroux V, Holtappels G, Pison C, Nadif R, Bousquet J, et al. Sensitization to staphylococcal enterotoxins and asthma severity: a longitudinal study in the EGEA cohort. Eur Respir J. 2019;54:1900198. Available from: http://erj.ersjournals.com/content/54/3/1900198.abstract

4. Profet M. The function of allergy: immunological defense against toxins. Q Rev Biol. 1991;66:23-62.

5. Galli SJ, Starkl P, Marichal T, Tsai M. Mast cells and IgE in defense against venoms: Possible "good side" of allergy? Allergol Int. 2016;65:3-15. Available from: http://dx.doi.org/10.1016/j.alit.2015.09.002

6. Starkl, P., Watzenboeck, M. L., Popov, L. M., Zahalka, S., Hladik, A., Lakovits, K., ... \& Knapp, S. (2020). IgE Effector Mechanisms, in Concert with Mast Cells, Contribute to Acquired Host Defense against Staphylococcusaureus. Immunity, 53(4), 793-804.7. Chen JS, Grassmann JDS, Gowthaman U, Olyha SJ, Simoneau T, Berin MC, et al. Flow cytometric identification of Tfh13 cells in mouse and human. J Allergy Clin Immunol. 2021;147(2):470-483. Available from: https://doi.org/10.1016/j.jaci.2020.04.063

8. Jiménez-Saiz R, Bruton K, Jordana M. Follicular $\mathrm{T}$ cells: From stability to failure. Allergy. 2020;75(4):1006-1007. doi: 10.1111/all.14167. Epub 2020 Feb 5. PMID: 31883377. 
9. Msallam R, Balla J, Rathore APS, Kared H, Malleret B, Saron WAA, et al. Fetal mast cells mediate postnatal allergic responses dependent on maternal IgE. Science. 2020;370:941-50.

10. Ferastraoaru D., Bax H.J., Bergmann C., Capron M., Castells M., Dombrowicz D., Fiebiger E., Gould H.J., Hartmann K., Jappe U., et al. AllergoOncology: Ultra-Low IgE, a potential novel biomarker in cancerA Position Paper of the European Academy of Allergy and Clinical Immunology (EAACI) Clin. Transl. Allergy. 2020;10:1-16. doi: 10.1186/s13601-020-00335-w.

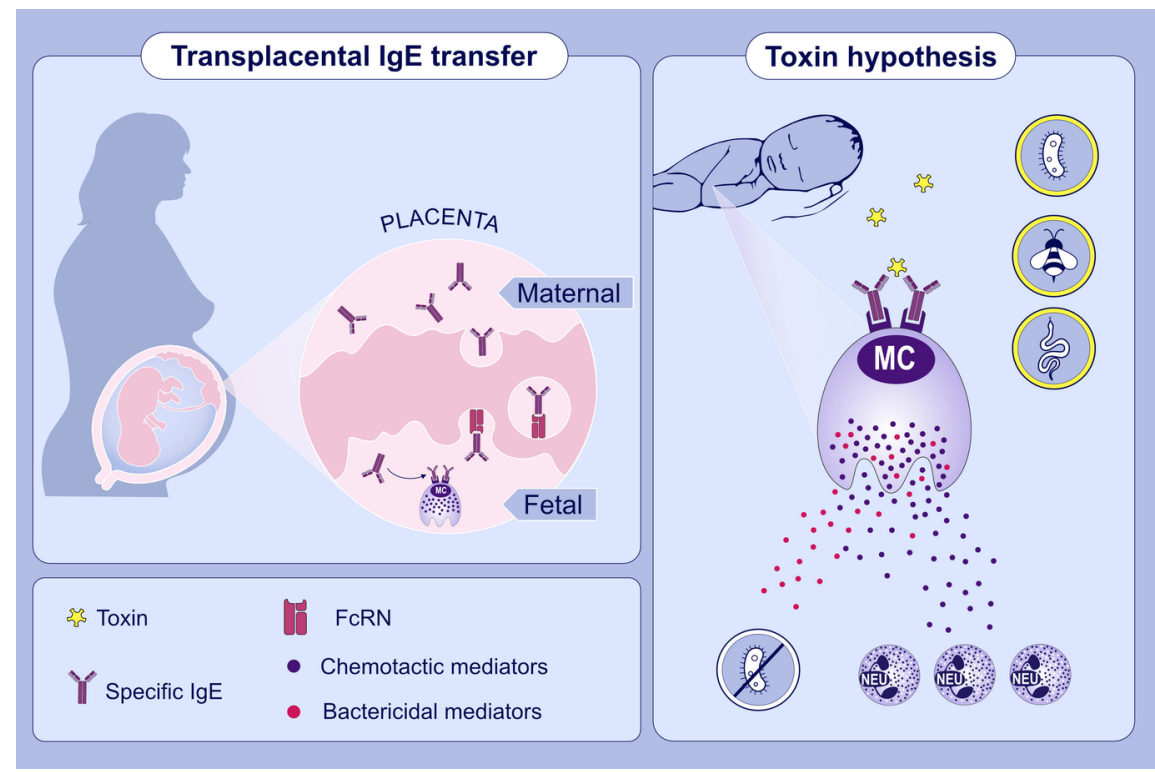

